of safranin to the phosphate eleuate produced a slow precipitate. This was separated on the centrifuge, suspended in water and the safranin removed with normal amyl alcohol. The aqueous solution was found to be infectious and contained no protein, phosphate or nitrogen.

As a control, sap from healthy plants was treated in exactly the same way as that from diseased plants. The behaviour of the mixed phosphate eleuate with acetone was quite different. Instead of the heavy white precipitate described above, a faint opalescence appeared which did not settle for many hours.

That plant viruses are not living organisms has been previously suggested. It has been stated that they are possibly enzymic in nature. Vinson and Petre are of the opinion that tobacco mosaic virus is of the nature of a simple protein. The isolation by us of a white crystalline compound which contains no nitrogen and yet is highly infectious appears to us to preclude the possibility of tobacco mosaic virus being of the nature of a living organism. In its precipitation with safranin it shows affinities with the proteolytic enzymes, but until we have made further investigation of the substance we can make no definite statement as to whether or not it is enzymic in nature.

E. BARTON-WRIGHT.

AlAN M. McBain.

Scottish Society for

Research in Plant Breeding.

Corstorphine, Edinburgh, 12.

${ }^{1}$ Contrib. Boyce Thompson Instit., 1, 479; 1929. 3, 131; 1931. See also Vinson, Phytopath., 23, 35; 1933.

\section{Wasting Disease of Eelgrass (Zostera marina)}

Is a report by Dr. Harrison F. Lewis of the Department of the Interior, Canada (referred to in NATURE of August 19, p. 277), it is mentioned that Dr. E. Silver Dowding has found in one blackened portion of the cortex of the rhizome "a coarse mycelium", "but as it could not be found in any other samples examined, it was not considered to be a cause of the disease". On account of this, I wish to communicate here that during last summer I have studied this disease, which now is widespread in Danish waters, and that I have always in the blackened spots seen the mycelium of a fungus as a constant feature.

I have examined principally leaves; only in a few cases have I seen infected rhizomes. I can say that I have never found a black spot without a mycelium. This mycelium consists of septate and branched threads a few $\mu$ broad, which when young are colourless, in the older stages dark brown. In the rhizomes it lives mostly in the outer cortex, in the leaves in the cells of the mesophyll, often growing for a long distance from cell to cell through the long air-spaces and creeping along the cell-walls. I have tried to isolate this fungus with the result that I have got a fungus of the group Hyphomycetes, which resembles the fungus growing in the leaves and in the rhizomes. As this pure culture has only been obtained once, I cannot say with certainty if the fungus $\mathrm{I}$ have isolated is identical with that of Zostera. Yet it is able, when growing in agaragar blocks in sea-water, to infect the leaves and produce the dark spots.

Another question is whother the Zostera fungus really is the cause of the disease ; it might be a saprophyte accompanying the disease caused by another organism such as a bacterium. The isolated mycelium produces conidia in great number in sea-water ; in the Zostera fungus I have only seen a few this summer.

In conformity with the foregoing, I believe that the observation of Dr. E. Silver Dowding concerning the occurrence of a mycelium in Canadian material deserves further investigation. In the coming year I hope to complete my own observations.

Henning E. Petersen.

Botanical Laboratory,

University of Copenhagen.

\section{Foundations of the New Field Theory}

The new field equations proposed recently ${ }^{1}$ can be derived from either of two principles, the first being a rather obvious physical statement, the other an equally obvious mathematical postulate.

(1) Einstein's mechanics is equivalent with the Lagrangian $m_{0} c^{2}\left\{1-\left(1-v^{2} / c^{2}\right)^{\frac{1}{2}}\right\}$. Historically, it has been derived from the idea of relativity ${ }^{2}$; but it could just as well have been found from experiments which show that electrons can not be arbitrarily accelerated. From this follows the existence of an upper limit for the velocity $c$; and the new Lagrangian is the simplest expression which is real only for $v<c$ and gives for the limit of small velocities the classical value $m_{0} v^{2} / 2$.

The problem of finding the exact law of the electromagnetic field can be attacked in a similar way. The classical Lagrangian $L=\frac{1}{2}\left(H^{2}-E^{2}\right)$ allows infinitely large values for the strengths of the field. But experience leads to the principle of the finite field. For the use of the classical function $L$ gives infinite values of self energy and other physical quantities which are, in fact, certainly finite. From this follows the existence of a limit of the field, $b$ (formerly called $\left.a^{-1}\right)$; and by the same reasoning as in mechanics, one constructs the new Lagrangian

$$
L=b^{2}\left[1-\left\{1-b^{-2}\left(E^{2}-H^{2}\right)\right\}^{\frac{1}{2}}\right]
$$

(2) The same result can be obtained by the mathematical postulate of the invariance of action.

Using the tensor notation, the classical Lagrangian is $L=\frac{1}{2} f_{k l} f k l$, where $f_{k l}=-f_{k l}$ represents the field $(H, E)$. The integral $\frac{1}{2} \int f_{k l} f^{k l} d \tau$ ( $d \tau$ element of space-time) is invariant for linear orthogonal, but not for general, transformations.

If $a_{k l}$ is any tensor and $\left|a_{k l}\right|$ its determinant, then $\int \sqrt{\left|a_{k l}\right|} d \tau$ is an invariant ${ }^{3}$. Now every tensor can be split up into a symmetrical and antisymmetrical part: $a_{k l}=g_{k l}+f_{k l} ; \quad g_{k l}=g_{l k}, f_{l k}=-f_{k l}$. The symmetrical part $g_{k l}$ should be identified with the metrical and $f_{k l}$ with the electromagnetic tensor. If we demand that the actions should be not only invariant, but should also take the form of the well-known expression $\frac{1}{2} \int f_{k l} f k l d \tau$ in the case of small electromagnetic fields and cartesian co-ordinate systems, we obtain

$$
L=\left(-\left|g_{k l}\right|\right)^{\frac{1}{2}}-\left(-\left|g_{k l}+f_{k l}\right|\right)^{\frac{1}{2}}
$$

This expression is entirely equivalent to the expression (1) for a statical field and a cartesian co-ordinate system. In the general (not statical) case an additional term, namely, $b^{-4}(E H)$ in the square root appears. One can get rid of this by choosing another but also invariant expression for $L$.

M. BorN.

L. INFELD.

1 Nature, 132, 282, Aug. 19, 1933; Proc. Roy. Soc., in the press. 2 Compare G. Levi-Civita, "Absolute Differential Calculus" (1927) Chap xi pp $280-301$.

Chap. xi, pp. $286-301$. "The Mathematical Theory of Relativity" (Cambridge, 1923), 107 . 